\title{
Application of Wearable Devices to Running during Training
}

\author{
Kai-Li Wang
}

\begin{abstract}
Wearable technology plays an important role in popularizing the sport of running. This is largely because wearable devices help runners' record data as a basis for gamification. However, today's gamification still relies heavily on point-based systems, and thus is considered too indirect to engage users who use wearable technology. To address this problem, the author of this study uses running as the case to examine routes and virtual data shared by runners (i.e. virtual sports data). The purpose is to achieve real-time gamification, which can also be applied to training runners. To explore how effective this method can be, the researcher held a test run. While running, the subjects wore a smartwatch imported with virtual sports data. This design created a benchmarking system, allowing the runners to compete with their virtual opponents. The experiment is designed to gain an insight into what may possibly affect this type of training (or virtual competition) model. A questionnaire survey was also administered to help improve this training model.
\end{abstract}

Index Terms-Wearable device, virtual sports data, gamification, running, technology acceptance model.

\section{INTRODUCTION}

\section{A. A Boost to the Popularity of Running}

Running in Taiwan has caught on in recent years. According to the frequently visited website Taipei Marathon, there were 84 running events held in 2006, exceeding 100 in 2010; in 2014, there were 432 events, which means more than one event each day. These figures bear witness to the local popularity of the sport. The reasons for this boom lie in the economic growth that drove the demand for healthy living, as well as the invention of ever-important wearable technology.

Wearable devices (also known as wearable technology) refer to smartwatches, sports bracelets, smart footwear, smart clothing, etc. Equipped with sensors, these devices track and record sports data. In the sport of running, a GPS-enabled watch or bracelet/wristband can monitor a runner's path. When used in conjunction with other data such as time, the gadget can further calculate pace in each segment of the runner's course. An accelerometer and a gyroscope enable gait to be recorded. A heartrate monitor allows a runner to measure his/her heartbeat. All this information can be used with height, weight and even age, to calculate calories burned. The final data can serve as a reference for athletic training or health management [1].

\section{B. Gamification}

The aforementioned method of data collection through

Manuscript received April 30, 2015; revised August 7, 2015.

K. L. Wang is with the National Taiwan Sport University, Taoyuan, 33301 Taiwan (e-mail: elverz@ ms8.hinet.net). wearable technology allows for more objective and accurate management of the user's health as an incentive for self-improvement. Wang [2] conducted focus interviews with athletes who used smartwatches. According to interviewees, accurate monitoring of running distance and time is crucial to setting athletic goals, and encouraging users to maintain their exercise habit. Thus, a system called Interactive Health Management Mechanism Based on Smart Watch was designed to satisfy an unmet demand. By accomplishing tasks designated by the system within a specific period of time, users of this system are granted points, which more deeply engage them in the sport [3].

This point-based system is actually an example of "gamification." According to Gartner Inc., an internationally acclaimed consultation company for the information application industry, gamification is a future trend. Gartner writes: "Gamification has emerged as a significant trend in recent years. Gamification uses game mechanics and game design techniques in non-gaming context - it's a powerful tool to engage employees, customers and the public to change behaviors, develop skills and drive innovation." [4] Gamification employs sports data collected by wearable devices to affect consumer behavior. This not only urges the public to exercise but also creates business opportunities [5].

Nike, the American sporting goods giant, followed suit early on by launching Nike+, a gamification-based app. The app counts walking/running paces. Moreover, it transforms sports data of all varieties into a point-based system called "NikeFuel." This allows users to measure athletic growth, and to encourage them to compete with friends for mutual improvement. Commercially, Nike+ is designed to market Nike products by taking advantage of user incentives.

\section{More Interactive Designs}

This kind of point-based system is the most well-known method of gamification [6]-[8]. But points may not suffice for a head-to-head contest. Therefore, designers of wearable devices and game-based apps are advised to develop a platform where players compete and rate their sports performances in a more direct way, rather than through an indirect point-based system. By this way, players can get the real-time feedback and more enjoyment [9].

As a sport, running offers a feasible case to apply this way of thinking. Most of today's wearable devices monitor running paths and sports data, such as location per second, instantaneous speed, and heartrate. Moreover, they can import the electronic data from users' previous routes or other people's data (hereinafter referred to as "virtual sports data"). The virtual sports data can be compared as a benchmark at any time with another user that ran the same route. Fig. 1 and Fig. 2 display the interface of a commercially available 
wearable device. Through gamification, users compare their athletic performances, feeling more motivated to exercise, either individually or competitively.

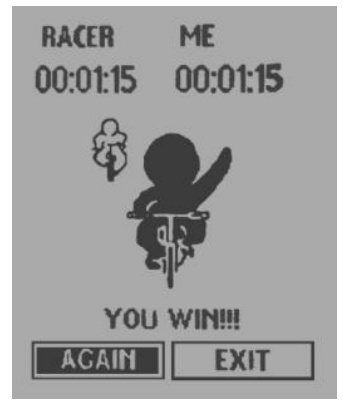

Fig. 1. Real-time comparison through a wearable device (win).

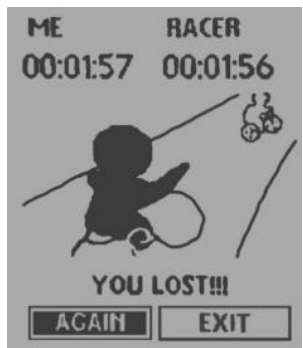

Fig. 2. Real-time comparison through a wearable device (loss).

The competition simulated by these devices will feel familiar to most runners. For example, in a marathon, runners closely follow runners ahead of them [10]. This strategy especially holds true during mid- or long-distance running events because runners can pace themselves more easily for better results. Even in non-competitive marathon events, organizers often arrange for pace-setters to run with participants. The goal is to help improve their athletic performances.

Most previous research on pacing was conducted from the perspective of exercise physiology, concluding that good pacing would elevate the cardiopulmonary function, rate of lactic acid metabolism, etc. [11], [12]. On the other hand, some studies on sports psychology indicate that a runner's well-developed, cognitive concept of pacing helps the runners set goals for a mid- or long-distance running event and better tolerate the burden [13]. However, little research explores the effect of pacing strategies on training. Only a few studies conclude that $\mathrm{PE}$ teachers found pacing more effective in training male students [14], [15].

To explore the effects of wearable technology in combination with gamification on training, running performance and subjects' feelings, 20 students participated in a trial run and then completed questionnaires as part of this study. The purpose is to understand how feasible the application of new technology is in sports training.

\section{MethodOlOGY}

\section{A. Research Design and Subjects}

The author of this study organized a small cross-country racing event on campus, with races ranging from $1 \mathrm{~km}$ to $3 \mathrm{~km}$ and $5 \mathrm{~km}$. Figs. 3, 4 and 5 display these routes. The event was designed as an experiment to determine whether inter-runner exchanges of virtual sports data could improve athletic performances. Subjects included students at National Taiwan Sport University, who completed a post-racing a questionnaire to share their thoughts and feelings about the event. Coupons and smartwatches were offered as an incentive to encourage more participants for higher rates of event participation and questionnaire response. Subjects enrolled by posting on campus bulletin boards, and screened according to their time of registration.

\section{B. Process}

Each of the 20 subjects ran the first $1-\mathrm{km}$ run for initial placement. The 20 runners were then ranked and cross-classified by place into two groups, each consisting of 10 people. One group ran $3 \mathrm{~km}$, the other $5 \mathrm{~km}$. All runners wore a smartwatch while running; their results (including routes) and ranking place were recorded as well.

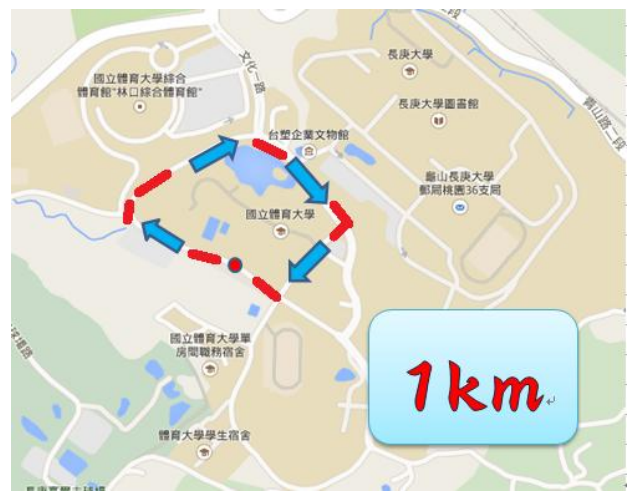

Fig. 3. The 1-km route.

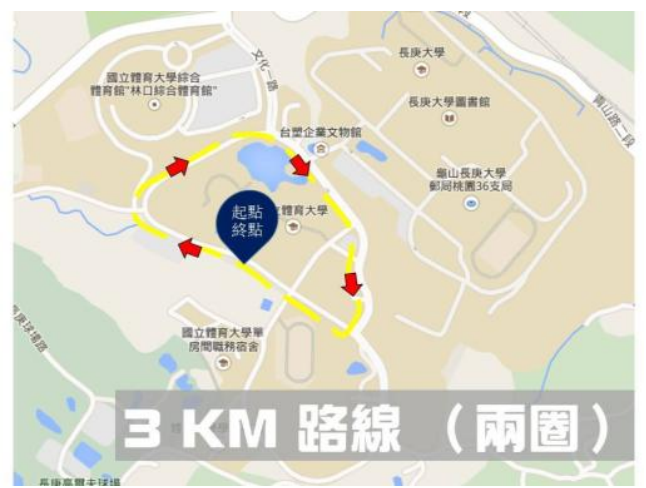

Fig. 4. The 3-km route (2 runs).

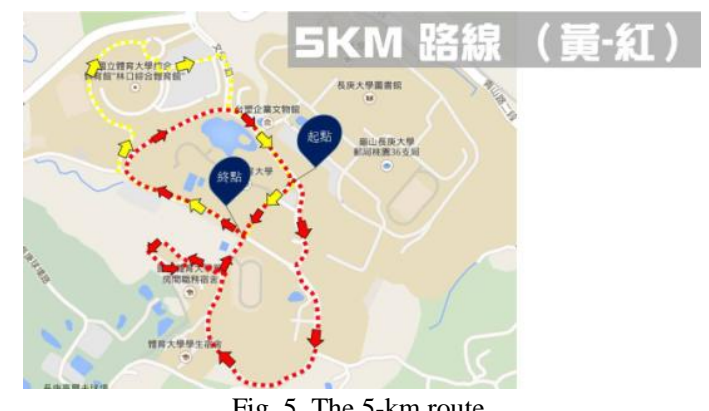

Fig. 5. The 5-km route.

After collecting the data, the researcher asked the subjects to switch routes. For example, the $3-\mathrm{km}$ group then ran the $5-\mathrm{km}$ route and the $5-\mathrm{km}$ group on the $3-\mathrm{km}$ route. To explore the effect of virtual sports data on runners, the researcher gave each runner a piece of virtual sports data taken from another runner ranked closely to him/her. The data was imported into 
the smartwatch. Then, the subjects ran again and were asked to pay attention to the data of their virtual opponents as displayed by the watch (shown in Figs. 1 and 2).

It is interesting to note that during the first run, some subjects misused the smartwatch and thus failed to generate running data. In addition, there were six subjects who had no virtual opponents ranked closely to them. According to the design of this experiment, they were asked to run again the same first route (i.e. the same distance) for their second run.

It is also worth mentioning that this study utilized two methods for selecting virtual sports data from closely ranked opponents. One method used the runner's virtual data directly corresponding to the rank from the two groups $\left(1^{\text {st }}\right.$ data matching model). The other method selected the runner's virtual sports data via the $1-\mathrm{km}$ route ranking $\left(2^{\text {nd }}\right.$ data matching model). In the experiment, seven subjects followed the first method, and another seven followed the second. For the control group, virtual sports data was not imported into the smartwatches of the remaining six subjects.

The researcher gave coupons worth NT $\$ 100$ to the subjects for each run. As a further incentive, the winners in both $3-\mathrm{km}$ and $5-\mathrm{km}$ groups, as well as the runners that made the most progress in each run, were given a smartwatch. The researcher also gave coupons worth NT\$50 to those who outpaced their virtual opponents. For runners without virtual opponents, they could be granted coupons worth NT\$50 if they ran faster on the second run (i.e. getting the better ranking).

\section{Questionnaire Survey}

The data of the subjects' athletic performances was collected in order to analyze the effect of virtual sports data on training. Furthermore, the subjects were surveyed with post-racing questionnaires asking how they felt about the event.

\section{ANALYSIS}

\section{A. Analyzing Sports Performances}

The researcher of this study aims to explore whether a benchmarking system based on the use of virtual sports data can contribute to sports performances. Due to budget concerns, only 20 subjects could participate, making a statistical test less meaningful. As such, this study uses descriptive statistics for further clarification.

Fig. 6 shows the win-to-loss ratio by group based on whether the runner had a virtual opponent for his/her second run. The so-called "win" means the runner outpaced their virtual opponents or ran faster on the second run (for runners without virtual opponents). Results show that seven runners did not know they could refer to the virtual data. One of them misused the data because he/she did not press the timing button, so his/her data was unavailable. Therefore, there are three groups: Virtual-Data group (winning ratio 50\%), No-Virtual-Data group (winning ratio $60 \%$ ), and Misusing-Virtual-Data group (winning ratio 33\%).

Some runners might intentionally run at a slower pace, due to a lack of confidence in their ability to win the prize. To solve this problem, the researcher ruled out the worst ten runners in the 1-km run, as shown in Fig. 7. The figure says that the winning ratio for the Virtual-Data group is $80 \%$, and that for the No-Virtual-Data group and the Misusing-Virtual-Data group is $33 \%$ and $0 \%$, respectively. It can be inferred that some runners felt more motivated to win the contest against their virtual opponents for the incentive.

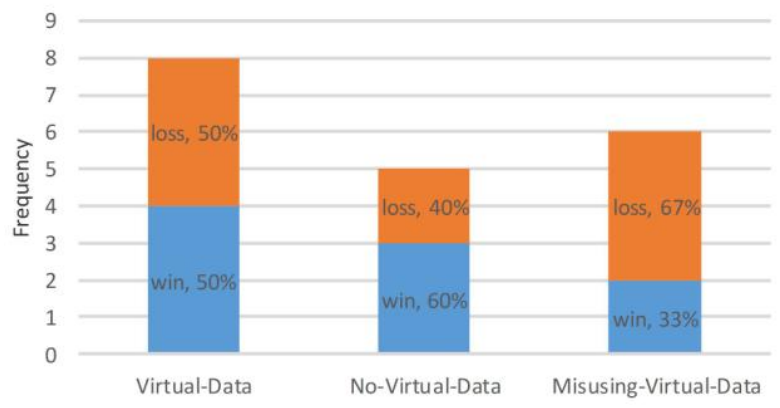

Fig. 6. Win-to-loss ratio by group of virtual data.

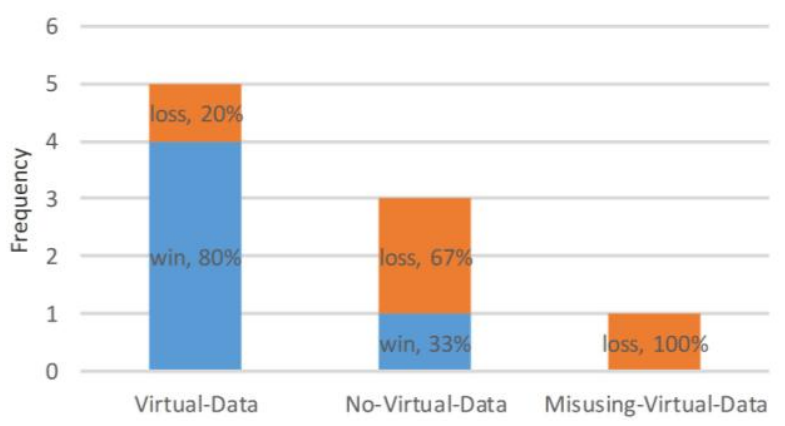

Fig. 7. Win-to-loss ratio by group of virtual data (for best 10 runners).

Fig. 8 explores the relationship between different virtual data matching models and running performance. The figure indicates that the winning ratio for the first matching model is $28.57 \%$, and that for the second model is $57.14 \%$. This is probably due to the first model's inaccuracy, leading the virtual sports data to be less effective. This reasoning, however, is no more than an assumption that does not statistically support the conclusion.

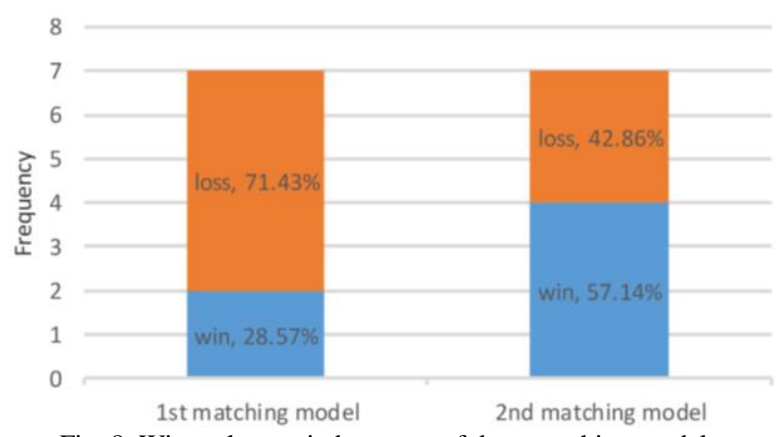

Fig. 8. Win-to-loss ratio by group of data matching model.

With regard to the difference in running performance between those who switched to the other group and those who did not, Fig. 9 shows a slight difference in the between-group winning ratio. Therefore, switching groups is presumably not an influential factor.

\section{B. How Subjects Felt}

The subjects were surveyed with questionnaires asking how they felt. One of the questions is "Choose at least one of the following options that describe how you felt about the contest against your virtual opponent." Fig. 10 shows the 
responses from runners aware of their virtual opponents in the second run. Most of them said "I felt more purposeful while running," with "I found the running more interesting" coming second, followed by "I found the running more exciting."

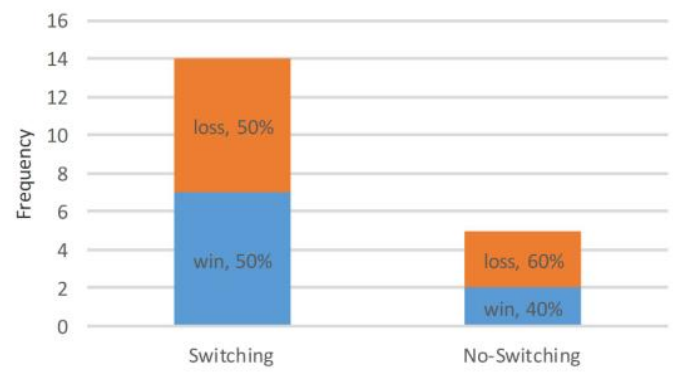

Fig. 9. Win-to-loss ratio by group of data matching model.

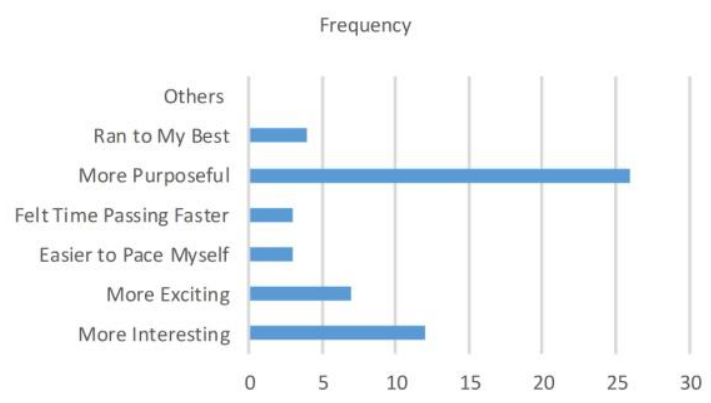

Fig. 10. How subjects felt about the running with virtual sports data.

Fig. 11 shows the statistics of the subjects' answers to the question "What do you find insufficient about the smartwatch that you used in the experiment based on your personal experience?" Most of them said "the interface is not intuitive," with "the device is bulky" coming second, followed by "the interface is complicated."

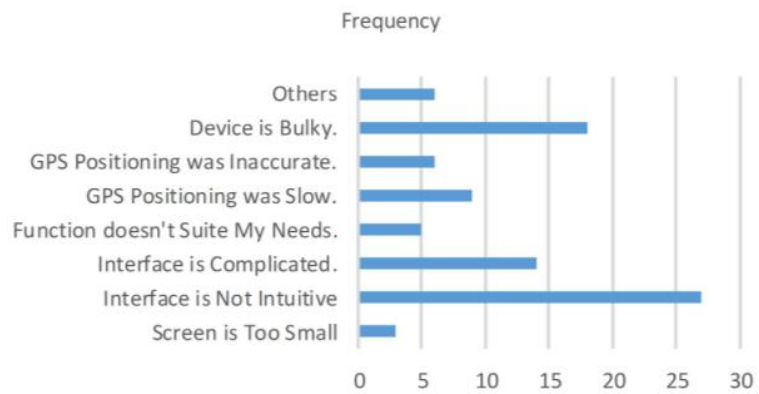

Fig. 11. Subjects' feeling about the insufficiency about the smartwatch.

This study also employed the concept of technology acceptance model (TAM) by asking subjects the following questions: "How useful do you think the smartwatch is in the experiment?", "How user-friendly do you think the smartwatch is in the experiment?" and "Will you consider using this kind of smartwatch in the future after trying the functions in the experiment?" The first question explores "perceived usefulness," the second "perceived ease of use," and the third "intention of use."[16] Table I shows the matrix for these questions. The subjects indicated that they might use this kind of product in the future when they find it useful, rather than ease of use. The product's usefulness bears little relationship to its ease of use. This is probably due to the subjects' inexperience in using the smartwatch and its complex interface. The finding also reflects the answers to first two questions above, which explore their subjective feelings.

TABLE I: THE RELATIONSHIP AMONG FACTORS OF TAM

\begin{tabular}{llll}
\hline \hline $\begin{array}{l}\text { Correlation } \\
\text { Coefficient }\end{array}$ & Usefulness & Ease of Use & Intention of Use \\
\hline Usefulness & 1 & 0.209 & 0.808 \\
Ease of Use & 0.209 & 1 & 0.505 \\
Intention of Use & 0.808 & 0.505 & 1 \\
\hline \hline
\end{tabular}

Lastly, Table II describes the subjects' answers to the question "How satisfied were you with your performance in the second run?"(i.e. self-efficacy) As it turned out, the Virtual-Data group scored the most in the self-efficacy (3.5 points), regardless of whether they won or lost. Then came the No-Virtual-Data + Win group (3.33 points), followed by the No-Virtual-Data + Loss group and the Misusing-Virtual-Data + Win group, both scoring 3 points. The Misusing-Virtual-Data + Loss group did the worst in the self-efficacy, posting only 2.5 points. These facts prove that the runners who knew they were competing with virtual opponents all exhibited better self-efficacy; others, less so.

TABLE II: THE CROSS TABLE OF SELF-EFFICACY

\begin{tabular}{lll}
\hline \hline Average Scores & Win & Loss \\
\hline Virtual-Data & 3.5 & 3.5 \\
No Virtual-Data & 3.33 & 3 \\
Misusing Virtual-Data & 3 & 2.5 \\
\hline \hline
\end{tabular}

\section{CONCLUSION AND SUGGESTIONS}

The researcher's conclusions and suggestions are as follows, according to the experiment and the questionnaire survey mentioned above.

1) Those who knew they were competing with virtual opponents scored better; those who didn't, less so. Moreover, the losers even felt a sense of inferiority (i.e. less self-efficacy). We can conclude that while training runners through a wearable device with gamification interface, the trainer is advised to familiarize themselves with the device/system. Otherwise, they may feel frustrated, leaving the expectation of increased performance unsatisfied.

2) The subjects who knew how to use the function/service found the training program interesting and exciting. This finding reflects the conclusion in previous research on exercise physiology and related studies. In this regard, future training models should develop more diversified and intriguing games for better efficiency and effectiveness.

3) The device's complex and less intuitive interface made it less user-friendly (i.e. ease of use). Therefore, more efforts are required for the development of similar devices to highlight the core value of the training model proposed in this study.

4) Another issue of concern lies in whether the virtual sports data matches the runner's competency. A virtual opponent at a different athletic level may render the training less effective. It is therefore advised to address 
this problem for similar models in the future.

\section{REFERENCES}

[1] W. C. Cheng, C. J. Cheng, W. C. Liu, and Y. W. Luo, "Design and implementation of sports health management system," Journal of Engineering Technology and Education, vol. 11, no. 1, pp. 14-23, March 2014.

[2] K. L. Wang, "A study on the use of means-end chain model to explore what values wearable devices bring to sport consumers," in Proc. SMAANZ Conference, 2014

[3] T. T. Tseng, "A study of interactive health management mechanism based on smart watch," M.S. thesis, Dept. of Innovation Design Engineering., National Kaohsiung First Univ. of Science and Technology, Kaohsiung, Taiwan, 2014.

[4] G. Bengsch, "Gamification: Engagement strategies for business and IT," Technology Special Report, Gartner, 2012.

[5] A. Inoue, Gamification, Taipei: Chinatimes, 2013.

[6] S. Nicholson, "A user-centered theoretical framework for meaningful gamification," presented at Games+Learning+Society 8.0, Madison, WI, June, 2012.

[7] J. McGonigal, Reality Is Broken: Why Games Make Us Better and How They Can Change the World, London: Penguin, 2011.

[8] G Zichermann and C. Cunningham, Gamification by Design: Implementing Game Mechanics in Web and Mobile Apps, Cambridge, MA: O'Reilly Media, 2011.

[9] J. H. Jung, C. Schneider, and J. Valacich, "Enhancing the motivational affordance of information systems: The effects of real-Time performance feedback and goal setting in group collaboration environments," Management Science, vol. 56, no. 4, pp. 724-742, 2010.
[10] C. P. Ho and J. J. Chao, "Application of tactics and strategies to midand long-distance running," Competitive Sport, vol. 12, no. 1, pp. 49-56, June 2010

[11] C. Y. Huang, "A comparative study of the lap split time for the superior runners in 2002 Soochow international ultra marathon 24hr Race," Hsiuping Journal of Humanities and Social Science, vol. 12, pp. 149-164, March 2009.

[12] L. M. Stirling, V. von Tscharner, P. F. Kugler, and B. M. Nigg, "Classification of muscle activity based on effert level during constant pace running," Journal of Electromyography and Kinesiology, vol. 21, pp. 566-571, 2011.

[13] K. Takai, "Cognitive strategies and recall of pace by long-distance runners," Perceptual and Motor Skills, vol. 86, pp. 763-770, 1998

[14] R. De Batea, Y. Zhangb, and S. H. Thompsonc, "Changes in commitment to physical activity among 8-to-11-year-old girls participating in a curriculum-based running program," American Journal of Health Education, vol. 38, no. 5, pp. 276-283, 2007.

[15] J. Denison, "Perspectives on coaching pace skill in distance running: A commentary," International Journal of Sports Science and Coaching, vol. 2, no. 3, pp. 217-238, 2007.

[16] F. Davis, "Perceived usefulness, perceived ease of use, and user acceptance of information technology," MIS Quarterly, vol. 13, no. 3, pp. 319-340, 1989

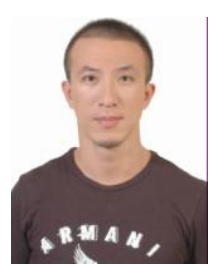

K. L. Wang works as an assistant professor in Graduate Institute of International Sport Affairs, National Taiwan Sport University. His area of interest is the law issue and public policy in leisure, entertainment and sport field. Recently, he did many researches about the image right, patent, public policy and legislation regarding the sport industry based on the approach of economics of law. 